\title{
Efficacy and safety of anti-angiogenic drugs combined with erlotinib in the treatment of advanced non-small cell lung cancer: a meta-analysis of randomized clinical trials
}

\author{
Zheng Chen ${ }^{1}$, Shu Jiang ${ }^{1}$, Xuechun $\mathrm{Li}^{1}$, Jingyao Zhang ${ }^{1}$, Liu Liu ${ }^{2}$, Juan $\mathrm{Li}^{2}$, Peiyu Cao ${ }^{2}$, Yong Xin ${ }^{1}$, \\ Longzhen Zhang ${ }^{1}$ \\ ${ }^{1}$ Department of Radiotherapy, Xuzhou Medical College Affiliated Hospital, Xuzhou, China; ${ }^{2}$ Cancer Institute, Xuzhou Medical University, Xuzhou, \\ China \\ Contributions: (I) Conception and design: Z Chen, S Jiang, X Li; (II) Administrative support: None; (III) Provision of study materials or patients: \\ None; (IV) Collection and assembly of data: Z Chen, S Jiang, X Li; (V) Data analysis and interpretation: All authors; (VI) Manuscript writing: All \\ authors; (VII) Final approval of manuscript: All authors. \\ Correspondence to: Yong Xin. Department of Radiotherapy, Xuzhou Medical College Affiliated Hospital, Xuzhou 221002, China. \\ Email: deep369@163.com; Longzhen Zhang. Department of Radiotherapy, Xuzhou Medical College Affiliated Hospital, Xuzhou 221002, China. \\ Email: jsxzzlz@126.com.
}

\begin{abstract}
Backgroundk To determine the efficacy and safety of anti-angiogenic drugs combined with erlotinib in the treatment of advanced non-small cell lung cancer (NSCLC), we performed a meta-analysis of 10 randomized controlled trials (RCTs).

Methods: An electronic literature search of PubMed, Cochrane Library, Embase, and other databases was performed. Literature was retrieved on December 2, 2019. We collected and compared RCTs on antiangiogenic drugs combined with erlotinib (A + E) for NSCLC, and analyzed outcomes including overall survival (OS), objective response rate (ORR), progression-free survival (PFS), and incidence of severe adverse events (grade $\geq 3$ AEs).

Results: Compared with the control group, combined treatment did not increase the ORR (RR 1.03, 95\% CI: 0.95-1.11, P=0.52) or prolong OS (HR 1.03, 95\% CI: 0.91-1.17, P=0.24); however, a significant improvement was seen in PFS (HR 0.63, 95\% CI: 0.57-0.70, $\mathrm{P}<0.001$ ). The subgroup analysis showed that patients with epidermal growth factor receptor (EGFR) mutations and patients with adenocarcinoma could benefit from combination therapy, as could those from Asian populations. Although combination therapy was found to carry an increased incidence of grade 3 or higher adverse events including diarrhea and proteinuria, these remained within controllable levels.
\end{abstract}

Conclusions: Anti-angiogenic drugs used in combination with erlotinib can significantly prolong PFS in patients with advanced NSCLC.

Keywords: Bevacizumab; ramucirumab; anti-angiogenic; erlotinib; non-small cell lung cancer (NSCLC)

Submitted Aug 16, 2020. Accepted for publication Dec 03, 2020.

doi: 10.21037/apm-20-1621

View this article at: http://dx.doi.org/10.21037/apm-20-1621

\section{Introduction}

Lung cancer remains the leading cause of death among cancer patients worldwide, and approximately $85 \%$ of lung cancers are non-small cell lung cancer (NSCLC) (1).

A series of clinical trials have demonstrated that epidermal growth factor receptor tyrosine kinase inhibitors (EGFR-TKIs) are associated with a higher tumor response rate, longer progression-free survival (PFS), and better quality of life compared to conventional chemotherapy. First-generation EGFR-TKIs, such as erlotinib, are the 
first-line treatment for advanced NSCLC (2-6). Although $60-80 \%$ of EGFR-positive tumor patients respond to these drugs, due to drug resistance, the median PFS rate is still low approximately 1 year) $(4,6-8)$. Osimertinib is a third-generation EGFR-TKI used to treat NSCLC and is generally well tolerated. In the FLAURA study, the PFS of osimertinib reached 18.9 [95\% confidence interval (CI) 15.2-21.4] months, and the updated result indicated that the overall survival (OS) reached 39 months (9). However, by analyzing the FLAURA study results, there was no statistically significant difference in the subgroups with or without exon 21 mutations. Furthermore, the OS of the Asian population did not exceed that of the European and American populations.

At present, it is still controversial whether osimertinib can replace erlotinib or gefitinib as the first-line treatment for patients with EGFR mutations. Therefore, the search for combination therapy is still of great importance to improving the survival of patients with advanced NSCLC. Anti-angiogenic drugs are mainly divided into endogenous angiogenesis inhibitors and indirect angiogenesis inhibitors. The indirect route features drugs targeting vascular endothelial growth factor (VEGF)/VEGF receptor (VEGFR), mainly including VEGF neutralizing antibodies and selective VEGFR-TKIs, such as sunitinib. In preclinical studies, compared with single inhibition of the EGFR pathway, the dual blocking of the EGFR and VEGF pathways was found to improve anti-tumor activity. Multiple clinical trials have shown that the combination of the VEGF-neutralizing antibody bevacizumab and EGFR-TKIs has a good effect, supporting the potential of dual pathway inhibition of EGFR and VEGF (10-15). Therefore, in this study, we explored the efficacy and safety of VEGF neutralizing antibodies, combined with erlotinib.

A meta-analysis of erlotinib combined with bevacizumab $(\mathrm{E}+\mathrm{B})$ was conducted by Zhou et al. (16); however, other VEGF neutralizing antibodies were not considered in their study, nor was the efficacy of the combination treatment evaluated for patients with brain metastasis. To address this knowledge gap, we conducted a meta-analysis of available clinical studies in the field of NSCLC. In this study, combination therapy with monotherapy was compared via a meta-analysis, and the objective response rate (ORR), OS, PFS, and the incidence of severe adverse events were examined. From the perspective of precision medicine, we also conducted subgroup analyses of the specific factors affecting PFS and severe adverse events. We present the following article in accordance with the PRISMA reporting checklist (available at http://dx.doi.org/10.21037/apm20-1621).

\section{Methods}

\section{Study selection}

The articles were screened by a researcher. The quality of the retrieved studies was assessed by two researchers independently, and data were extracted and cross-checked in accordance with the systematic review and meta-analysis (PRISMA) guidelines (17). Disagreements between the two researchers were resolved by negotiation after discussion with the entire team.

\section{Search strategy}

On 2 December 2019, a literature search was performed of electronic databases including PubMed, Embase, Web of Science, and the Cochrane Library, as well as several international conference databases including the American Society of Clinical Oncology, the European Society of Medical Oncology, European Cancer Conference, and the World Lung Cancer Conference. The focus of the literature search was published randomized controlled trials (RCTs) of combinations of anti-angiogenic drugs and erlotinib for the treatment of NSCLC. The keywords were "Non-small cell lung cancer" (MeSH), "Erlotinib”, "Anti-angiogenesis", "Ramucirumab", "Bevacizumab".

The inclusion criteria were as follows: (I) Published RCTs; (II) the experimental group was A + E combination therapy; (III) the anti-angiogenic drugs were monoclonal antibodies; (IV) patients with advanced-stage (IIIb/IV/ relapsed) NSCLC confirmed histologically or cytologically; (V) patients were aged 18 years or above; (VI) the language of the included documents was English; (VII) at least one of the clinical outcome indicators (ORR, PFS, or OS) was reported in the trial; (VIII) grade 3-5 adverse events or specific adverse events identified and graded by the National Cancer Institute's common terminology criteria for adverse events.

The exclusion criteria were as follows: (I) The drug was not approved by any regulatory agencies; (II) known EGFR Thr790Met mutation; (III) research on animals or cadaver studies; (IV) studies where data was unavailable or studies that did not have valid data; Meta-analyses, case reports, and retrospective reports. 


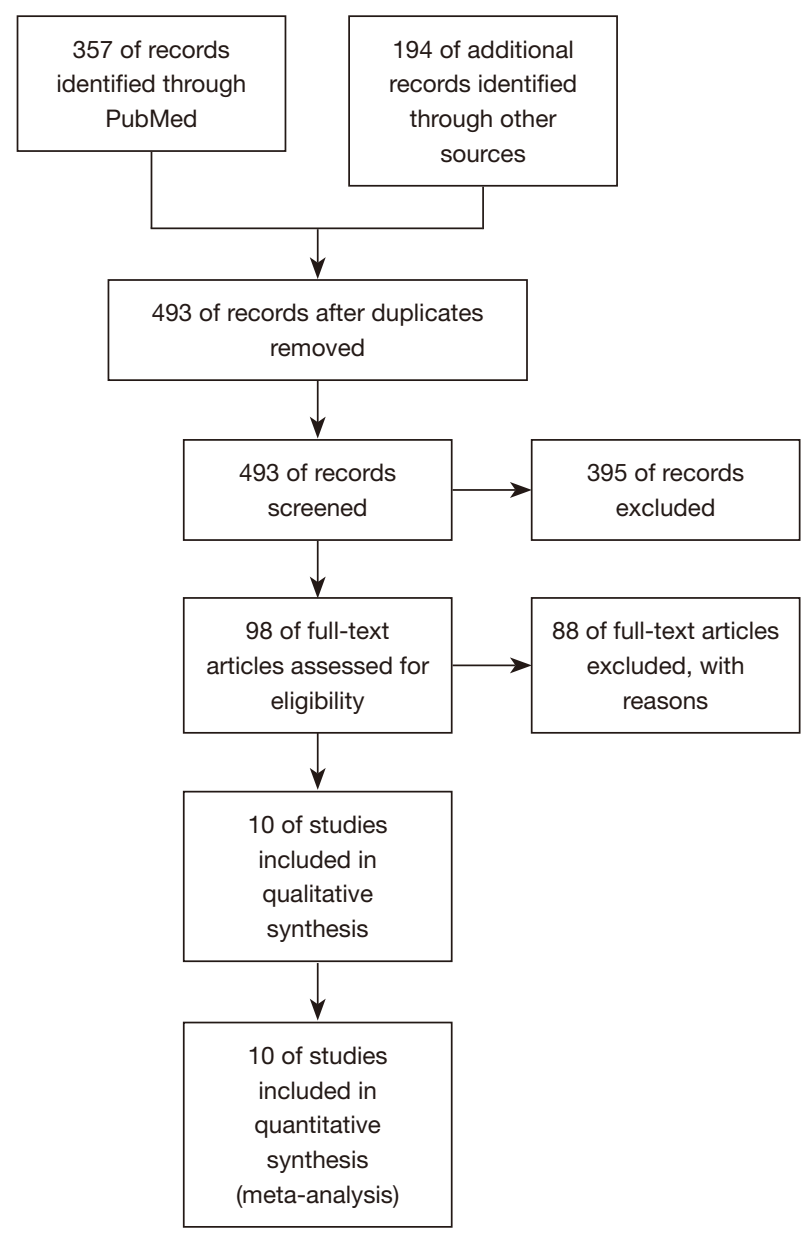

Figure 1 Flowchart of literature screening.

\section{Data extraction}

Patient baseline data were independently extracted from RCTs that met the inclusion criteria by the two researchers. The anti-angiogenic monoclonal antibodies included bevacizumab and ramucirumab. The extracted data included the authors of the article, year of publication, number of enrolled patients, sex ratio, race, histology, EGFR status, and the ratio of brain metastases (Table 1).

\section{Quality evaluation}

The two researchers used the Cochrane Handbook for Systematic Reviews of Interventions $(26,27)$ to assess the risk of bias. Using the Cochrane Collaboration tool, every study has a high, low, or unclear risk of bias. If the two researchers differed in their interpretation, the disagreement was resolved through discussion.

\section{Outcome measures}

(I) PFS: the time from randomization to tumor progression or death;

(II) OS: the time from randomization to death, which is considered the best treatment endpoint in cancer clinical trials;

(III) ORR: the proportion of patients whose tumors shrank to a certain degree and remained unchanged for a while, including cases of complete remission (CR) and partial remission (PR);

(IV) Subgroup analysis of PFS: the purpose of this metaanalysis was to explore the efficacy and safety of combined medications, and simultaneously to conduct a subgroup analysis of PFS to find the population that could benefit from these treatments. Specifically, the subgroup analysis was performed to determine the effects of pathology, race, the presence or absence of EGFR mutation, mutation status, and the presence or absence of brain metastasis on the PFS results;

(V) The incidence of grade 3-5 adverse events (rash, diarrhea, hypertension, proteinuria, fatigue, hemorrhage, and fatigue) were analyzed.

\section{Statistical analysis}

Statistical analysis was performed using RevMan version 5.3. Heterogeneity was assessed using the $\mathrm{I}^{2}$ statistic. If significant heterogeneity was detected $\left(\mathrm{I}^{2}>50 \%\right.$ or $\left.\mathrm{P}<0.01\right)$, the random-effects model was used, otherwise the fixedeffects model was used. Time-event variables, including OS and PFS, were evaluated based on hazard ratio (HR) with $95 \%$ CI. Dichotomous variables, such as ORR and the incidence of adverse events, were estimated using the risk ratio (RR). The forest plot lists the hypothesis test results for each variable. A sensitivity analysis was performed on the outcome indicators with significant heterogeneity, and each included study was excluded to determine the source of heterogeneity.

\section{Literature search}

A total of 10 studies were retrieved from 551 articles that met our initial inclusion criteria. 57 duplicate studies and 395 unqualified studies were removed. After the full text had been read, 88 of the remaining articles were excluded. Finally, 10 studies were included in the meta-analysis (Figure 1). An assessment of article quality is shown in Figure 2. 


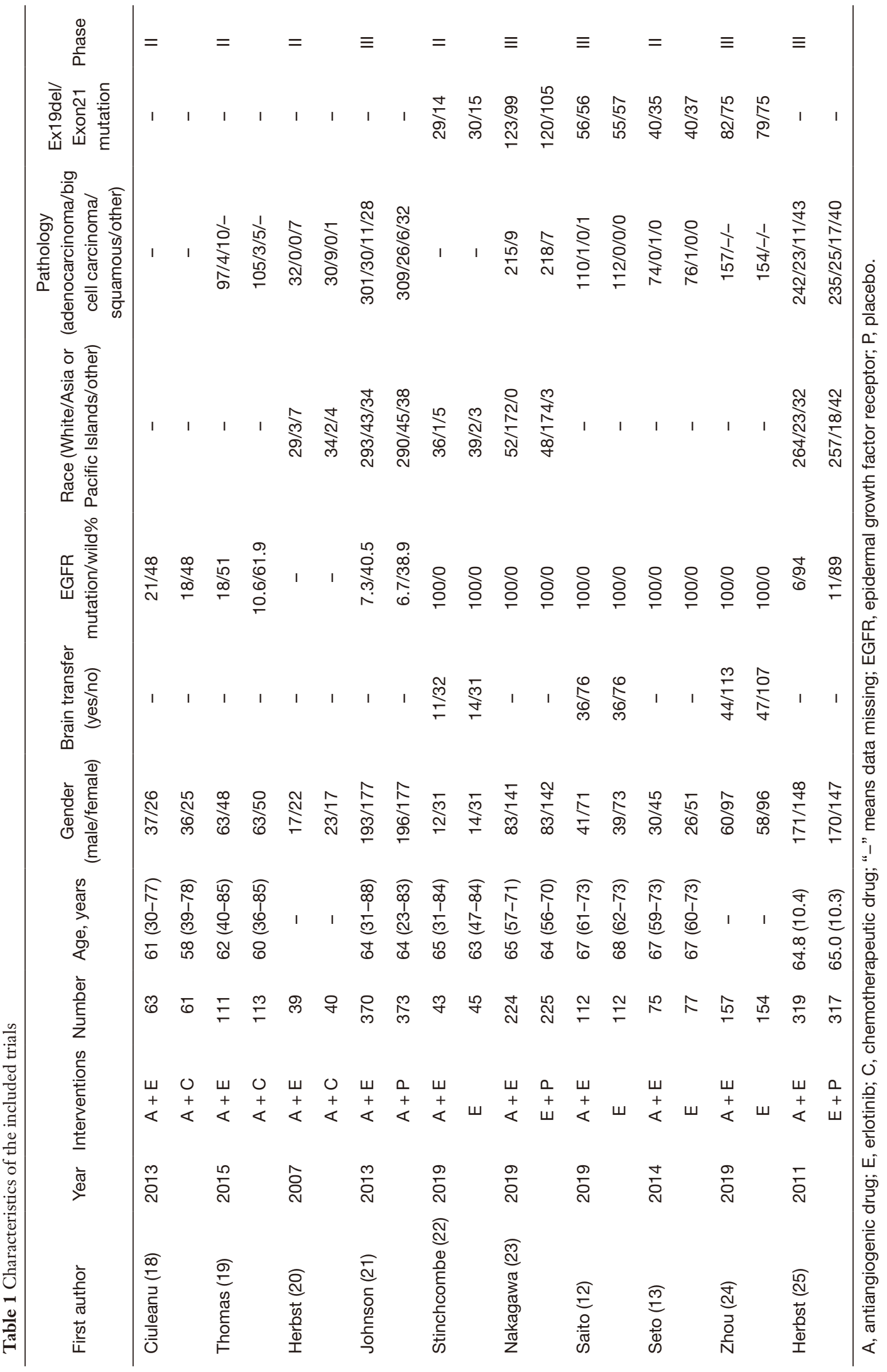




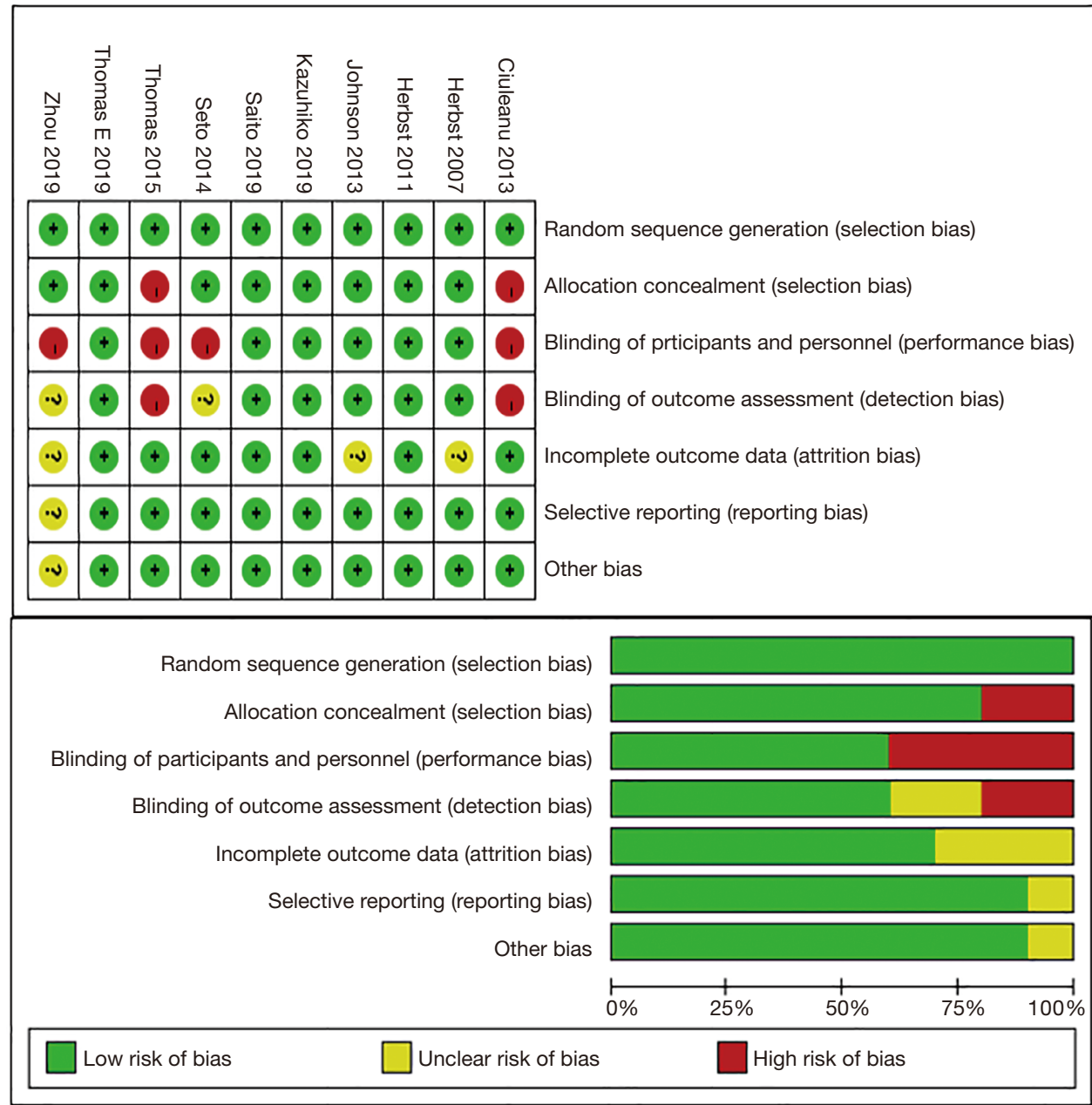

Figure 2 The assessment results of the risk of bias for included trails.

\section{Experimental groups}

$\mathrm{A}+\mathrm{E}$ was the combined group, and $\mathrm{E} / \mathrm{E}+\mathrm{P} / \mathrm{A}+\mathrm{C} / \mathrm{A} / \mathrm{A}+\mathrm{P}$ were the control groups.

\section{Results}

\section{Outcome measures}

\section{PFS}

All 10 studies reported PFS (Figure 3A). Studies by Ciuleanu et al. (18) and Thomas et al. (19) resulted in significant heterogeneity $\left(\mathrm{I}^{2}=86.1 \%\right)$ and were removed after sensitivity analysis. After removal, $\mathrm{I}^{2}$ was reduced to $0 \%$, allowing the use of the fixed-effects model. In the group of $\mathrm{A}+\mathrm{E} v s . \mathrm{E} /$ $\mathrm{E}+\mathrm{P}$ and $\mathrm{A}+\mathrm{E} v s . \mathrm{A} / \mathrm{A}+\mathrm{P}, \mathrm{PFS}(\mathrm{HR} 0.61,95 \% \mathrm{CI}: 0.55-$ $0.69, \mathrm{P}<0.001$; HR 0.68, 95\% CI: 0.56-0.82, $\mathrm{P}<0.001$ ). However, in the group of $\mathrm{A}+\mathrm{E} v s . \mathrm{A}+\mathrm{C}$, PFS (HR 0.72,
95\% CI: $0.42-1.23, \mathrm{P}=0.23)$, combination therapy did not significantly improve PFS.

\section{OS}

A total of six studies $(18-22,25)$ reported OS (Figure 3B). Because of the low heterogeneity ( $=26 \%$ ), the fixed-effects model was used. The results showed that there was no significant difference in OS between the combined group and the control group (HR 1.03, 95\% CI: 0.91-1.17, $\mathrm{P}=0.63$ ).

\section{ORR}

A total of eight studies $(12,13,18-20,22,23,25)$ reported the ORR (Figure 3C). Studies by Herbst et al. (25) and Thomas et al. (19) resulted in significant heterogeneity and were removed after sensitivity analysis. The fixed effects model was used because $\mathrm{I}^{2}<50 \%$ after removal. In the $\mathrm{A}+\mathrm{E} v s . \mathrm{E} / \mathrm{E}+\mathrm{P}$ and $\mathrm{A}+\mathrm{E} v s . \mathrm{A}+\mathrm{C}$ comparisons, combination therapy was 


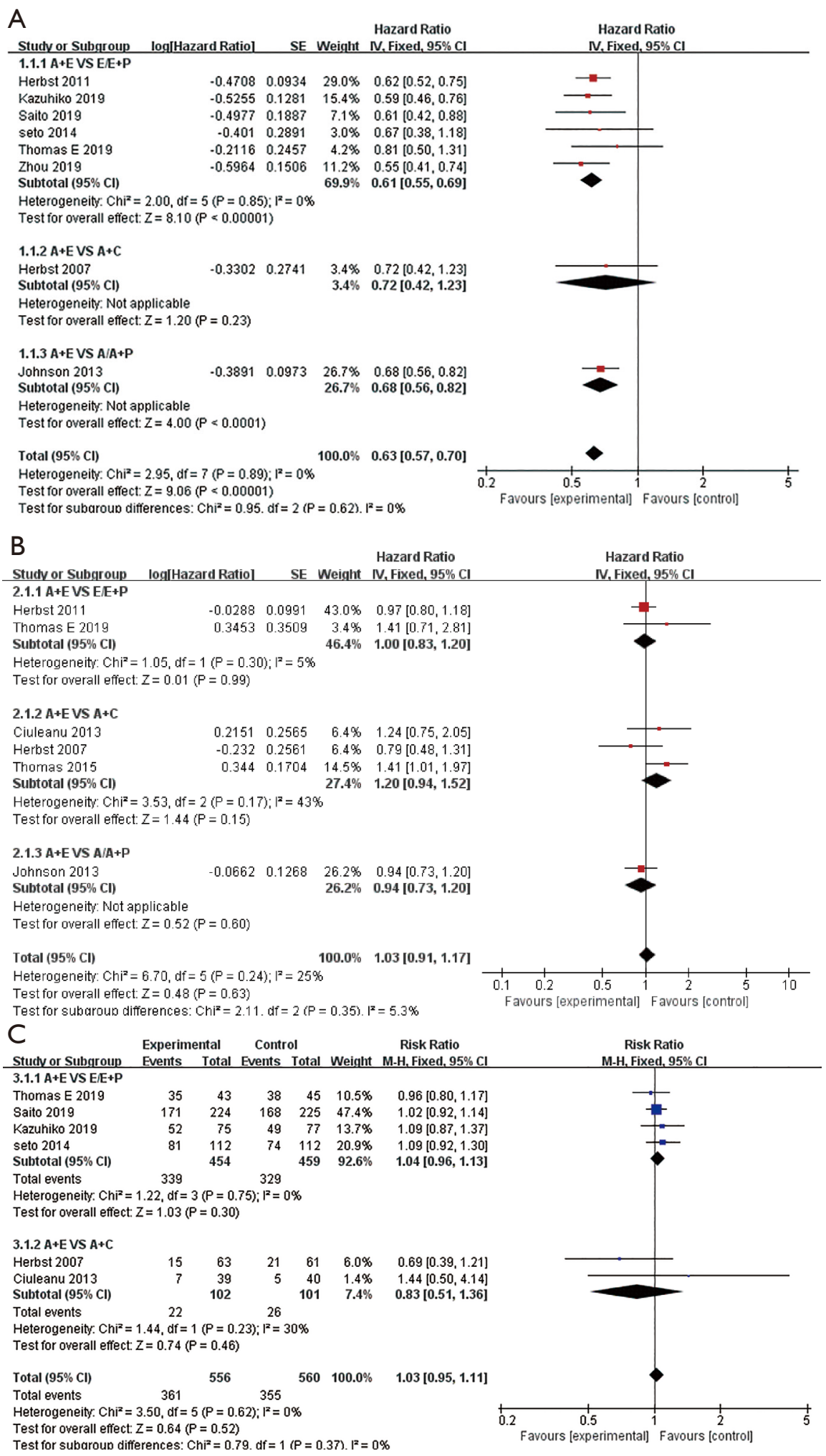

Figure 3 The forest plot of time-event variables (PFS, OS) and dichotomous variables (ORR, the incidence of adverse events). (A) PFS for combination therapy of anti-angiogenic drugs plus erlotinib. $(\mathrm{B}, \mathrm{C}) \mathrm{OS}$, ORR for combination therapy of anti-angiogenic drugs plus erlotinib. 
not found to be associated with elevated ORR (RR 1.04, 95\% CI: $0.96-1.13, \mathrm{P}=0.30$; RR 0.83, 95\% CI: $0.51-1.36, \mathrm{P}=0.46$ ).

\section{PFS in the subgroup analysis}

A total of eight studies (12-13,19,21-25) reported PFS data on EGFR mutations (Figure $4 A$ ). Compared with the control group, combination therapy significantly prolonged the PFS of patients with EGFR mutations (HR 0.60, 95\% CI: $0.52-0.70 ; \mathrm{P}<0.001)$. In four studies $(18,19,21,25)$, there was no statistically significant difference in PFS (HR 1.18, 95\% CI: $1.00-1.39 ; \mathrm{P}=0.05$ ) between the combined group and the control group in EGFR wild-type patients.

A total of three studies $(12,21,24)$ reported brain metastasis subgroup data (Figure 4B). In the combined treatment group, patients with brain metastases (HR 0.59, 95\% CI: $0.40-0.87 ; \mathrm{P}=0.007)$ and without brain metastases (HR 0.66, 95\% CI: 0.57-0.77; $\mathrm{P}<0.001$ ) had significantly extended PFS.

A total of three studies $(21,23,25)$ reported subgroup data on race (Figure $4 C$ ). Combination treatment significantly prolonged the PFS of Asian patients (HR 0.68, 95\% CI: 0.58-0.80; $\mathrm{P}<0.001)$, while European and white American patients (HR 0.93, 95\% CI: 0.77-1.12; $\mathrm{P}=0.44$ ) showed no statistical differences in PFS between the two groups.

A total of four studies $(12,13,23,24)$ reported subgroup data on EGFR mutation types (Figure 4D). In the combined treatment group, the PFS of patients with EGFR exon 19 deletion mutations (HR 0.61, 95\% CI: 0.49-0.75; $\mathrm{P}<0.001$ ) and exon 21 Leu858Arg mutations (HR 0.59, 95\% CI: $0.47-0.73 ; \mathrm{P}<0.001$ ) was significantly prolonged.

Several studies reported subgroup data for pathological type (Figure 4E). PFS was significantly prolonged in patients with adenocarcinoma $(13,21,24,25)$ (HR 0.73, 95\% CI: 0.64-0.83; $\mathrm{P}<0.001)$. However, for patients with large cell carcinoma (21,25) (HR 0.70, 95\% CI: 0.43-1.14; $\mathrm{P}=0.15$ ), squamous cell carcinoma $(21,25)$ (HR 1.02, 95\% CI: 0.48-2.13; $\mathrm{P}=0.97)$, or other diseases (21,25) (HR 0.88, 95\% CI: 0.99-1.33; P=0.56), combination therapy did not significantly prolong PFS.

\section{Incidence of serious adverse reactions}

Grade 3-5 adverse events with a high incidence, such as hypertension $(12,13,20-25)$, rash $(12,13,18-23,25)$, proteinuria $(12,13,18,21-24)$, diarrhea $(12,13,18-23)$, hemorrhage $(12,13,19,25)$, fatigue $(13,18-20,22)$, and dyspnea $(18-20,22)$, were selected. The results are shown in Figure 5. Compared with the control group, the combined treatment group did not show a significant difference in terms of rashes (RR 1.80, 95\%
CI: 0.84-3.85; $\mathrm{P}=0.13$ ) (Figure $5 A$ ), fatigue (RR 0.57, 95\% CI: $0.28-1.18 ; \mathrm{P}=0.13$ ) (Figure 5B), hemorrhage (RR 1.22, 95\% CI: 0.53-2.79; $\mathrm{P}=0.64$ ) (Figure 5D), hypertension (RR 1.82, 95\% CI: 0.81-4.09; $\mathrm{P}=0.15$ ) (Figure 5F), and dyspnea (RR 1.11, 95\% CI: 0.58-2.14; $\mathrm{P}=0.75$ ) (Figure $5 G$ ). However, the two groups differed significantly in terms of proteinuria (RR 4.83, 95\% CI: 1.63-14.31; $\mathrm{P}=0.004$ ) (Figure 5C) and diarrhea (RR 2.37, 95\% CI: 1.29-4.35; $\mathrm{P}=0.005$ ) (Figure 5E).

\section{Discussion}

Our meta-analysis showed that combination therapy significantly prolonged PFS in patients with advanced NSCLC compared with monotherapy. However, combination therapy was not found to prolong ORR or OS. Our subgroup analysis showed that prolonged PFS was significantly correlated with the presence of EGFR mutation, the pathological diagnosis of adenocarcinoma, and Asian race. Therefore, genetic diagnosis before treatment is essential. In the comparison between the $\mathrm{A}+\mathrm{E}$ vs. A + C group, Ciuleanu et al. (18) and Thomas et al. (19) added heterogeneity, which was removed from the statistics in Figure $3 a$ through sensitivity analysis. The PFS of patients with EGFR mutations in Thomas et al. was (HR 0.91, 95\% CI: $0.42-1.98)$, the PFS of wild-type patients was (HR 2.07, 95\% CI: 1.42-3.02). In Ciuleanu et al., the PFS of EGFR wild-type patients was (HR 2.08, 95\% CI: 0.98-4.40). All results were included in the subgroup analysis, which was consistent with the conclusion that combined treatment can significantly prolong the PFS of patients with EGFR mutations. Ciuleanu et al. suggested that the reason why erlotinib was not effective in patients with EGFR mutations might be due to the small number of groups, which leads to a failure to obtain PFS. Whether the $\mathrm{A}+\mathrm{C}$ scheme can prolong the PFS of EGFR wildtype patients requires a large number of RCTs. Previous studies have shown that EGFR-TKI treatment had a better effect in patients with exon 19 deletion mutations than in patients with exon 21 mutations $(4,5,28)$, and our research suggests that combination therapy has similar effects on the two mutations. Combination therapy is therefore a novel treatment direction for patients with mutations to exon 21. Brain metastasis is a well-known poor prognostic factor for NSCLC disease progression. Our meta-analysis suggests that combination therapy is equally as effective for patients with brain metastases.

In this study, grade 3-5 adverse events with a high incidence were selected for analysis. Combination therapy 


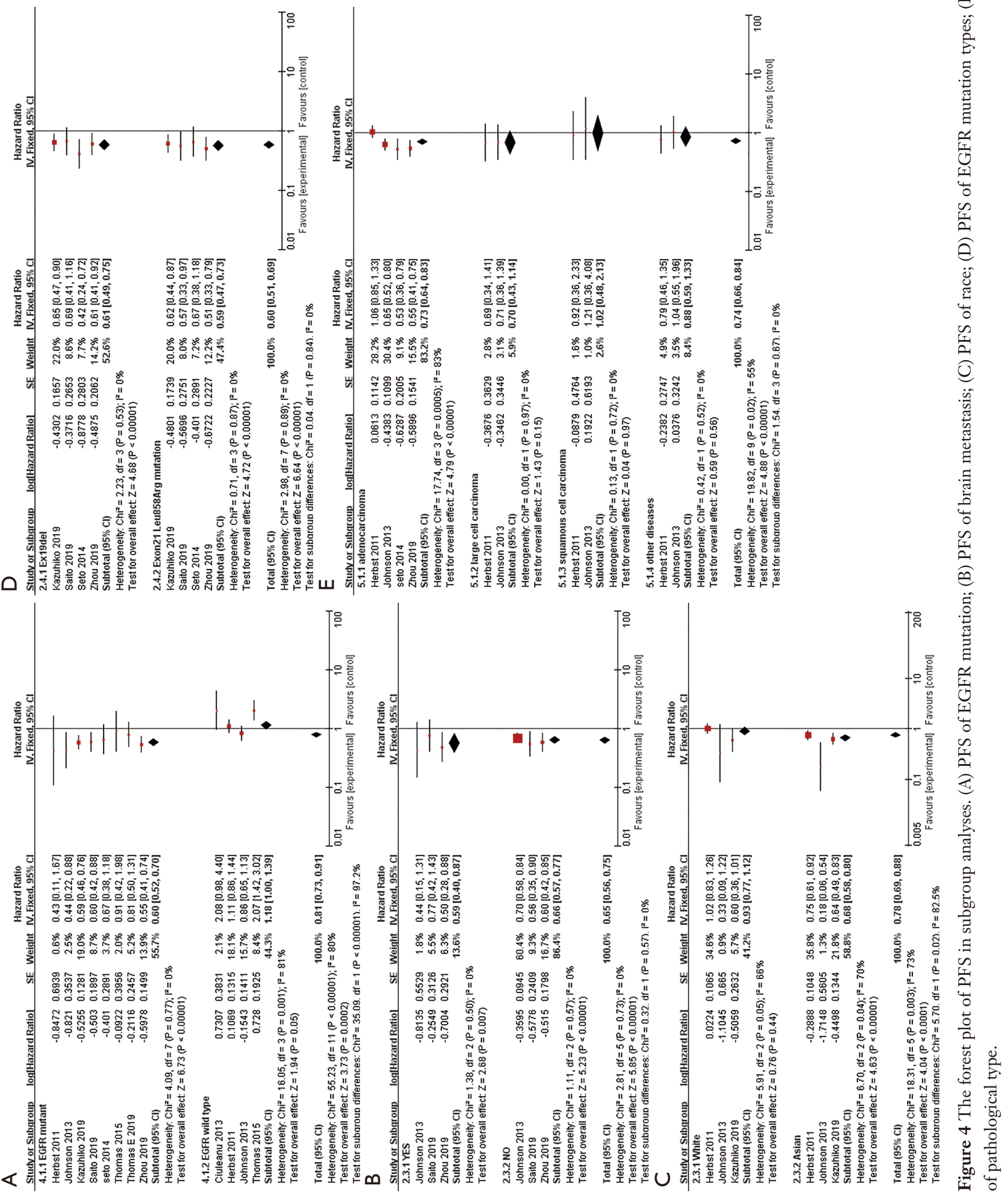




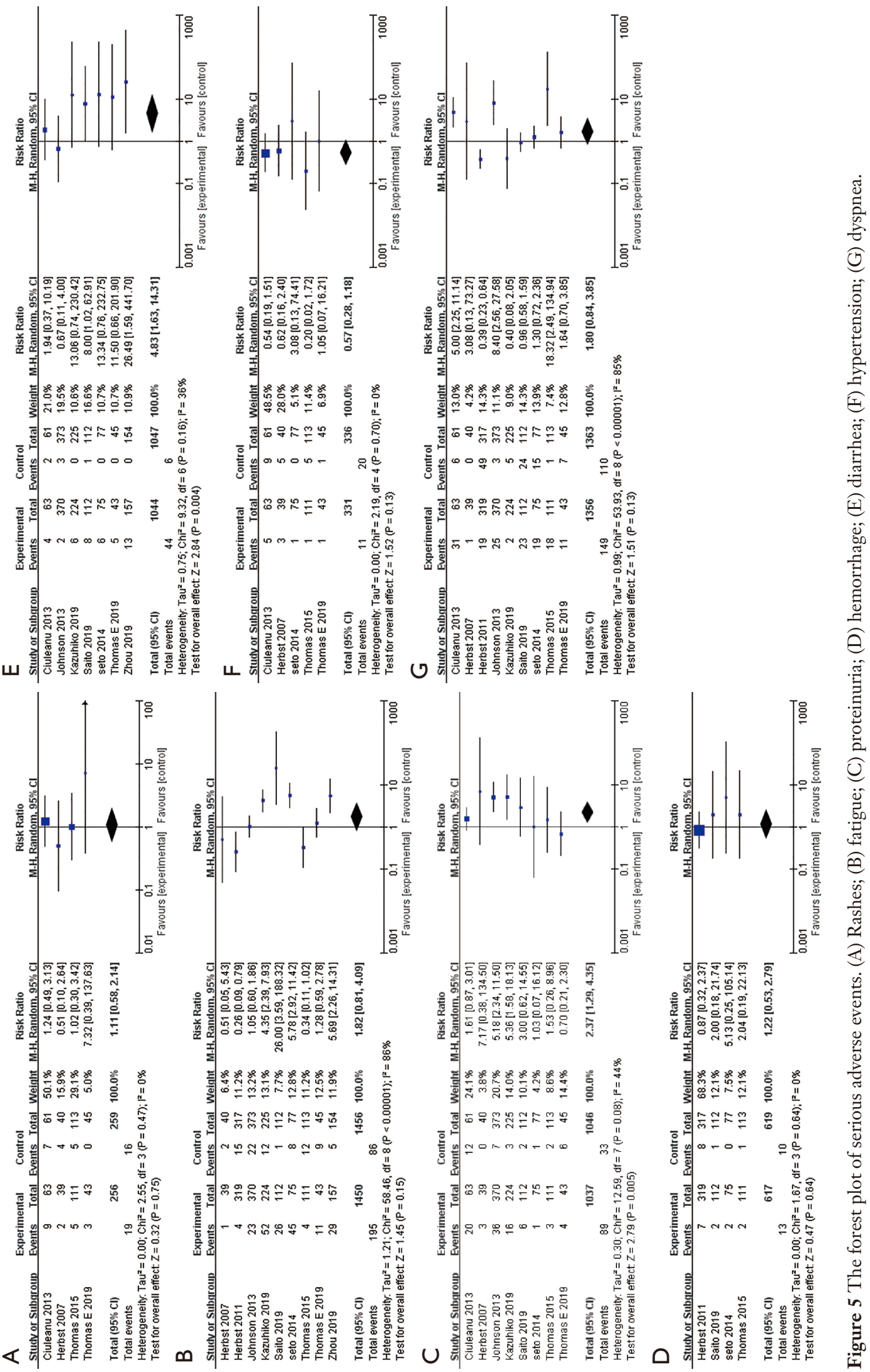


increased the incidence of diarrhea and proteinuria, while the rates of hypertension, rash, dyspnea, hemorrhage, and fatigue were not significantly higher than those in the control group. These results suggest that combination therapy increases only the incidence of minor adverse events, which can be treated with appropriate protection of renal function and antidiarrheal medication. In clinical use, the importance of individual reactions to drug combinations should be considered, and prompt symptomatic treatment should be given. In assessing the appropriateness of treatment, the cost of combination therapy and monotherapy should also be an important consideration.

The most common mechanism of resistance in first- and second-generation EGFR-TKIs is the Thr790 mutation, accounting for $50-60 \%$. Thr790 mutation reduces the ability of reversible EGFR-TKIs to bind to the EGFR ATP-binding pocket, thus reducing EGFR-TKI-mediated downstream signaling inhibition $(29,30)$. The RELAY study showed that the combination group and the control group had a similar incidence of Thr790Met after drug resistance $(\mathrm{P}=0.492)$. Furthermore, in an experiment by Zhou et al. (24), the incidence of Thr790Met after resistance using the A+T (EGFR-TKI) treatment regimen was $33 \%$, and in the $\mathrm{T}$ single-drug group, the incidence was $42 \%$. The data also confirmed that the addition of anti-angiogenic drugs to erlotinib did not prevent the emergence of Thr790Met corrective mechanisms. Subsequent use of osimertinib as the next line of treatment is still a viable treatment option.

\section{Advantages and limitations}

The main advantage of this study is that we conducted a comprehensive search of databases and literature, including research on conference coverage. Secondly, we conducted a detailed stratified analysis of subgroups and adverse events, and added subgroups on the type of EGFR mutation and the presence or absence of brain metastases. This can aid in clinically screening out patients most likely to benefit from treatment.

A major limitation of our study is that there was only one RCT of ramucirumab, and our conclusions need to be confirmed with larger sample sizes and high-quality clinical studies. Secondly, four RCTs were open-label studies, which might have introduced bias.

\section{Conclusions}

In summary, the results of this meta-analysis showed that combination therapy could improve PFS in patients with EGFR mutations and patients with adecarcinoma, as well as those from Asian populations. It also increased the incidence of grade 3 and above adverse events including diarrhea and proteinuria, although these complications remained within the controllable range. Therefore, we recommend combination therapy for the patients described above. Our findings help to resolve existing controversies and highlight the benefits of combination therapy, which may thereby help to improve personalized treatment options.

\section{Acknowledgments}

We appreciate all the support of our colleagues, friends, and experts from Xuzhou Medical College Affiliated Hospital.

Funding: This work was supported by the Project of Scientific Research and Practical Innovation Plan for Postgraduates in Jiangsu Province, China (SJCX19_0765), National Natural Science Foundation of China (Grant No. 81972845) and Natural Science Foundation of the Jiangsu Higher Education Institutions of China (Grant No. BK20190989).

\section{Footnote}

Reporting Checklist: The authors have completed the PRISMA reporting checklist. Available at http://dx.doi. org/10.21037/apm-20-1621

Conflicts of Interest: All authors have completed the ICMJE uniform disclosure form (available at http://dx.doi. org/10.21037/apm-20-1621). The authors have no other conflicts of interest to declare.

Ethical Statement: The authors are accountable for all aspects of the work in ensuring that questions related to the accuracy or integrity of any part of the work are appropriately investigated and resolved.

Open Access Statement: This is an Open Access article distributed in accordance with the Creative Commons Attribution-NonCommercial-NoDerivs 4.0 International License (CC BY-NC-ND 4.0), which permits the noncommercial replication and distribution of the article with the strict proviso that no changes or edits are made and the original work is properly cited (including links to both the formal publication through the relevant DOI and the license). See: https://creativecommons.org/licenses/by-nc-nd/4.0/. 


\section{References}

1. Midha A, Dearden S, McCormack R. EGFR mutation incidence in non-small-cell lung cancer of adenocarcinoma histology: a systematic review and global map by ethnicity (mutMapII). Am J Cancer Res 2015;5:2892-911.

2. Wu YL, Zhou C, Liam CK, et al. First-line erlotinib versus gemcitabine/cisplatin in patients with advanced EGFR mutation-positive non-small-cell lung cancer: analyses from the phase III, randomised, open-label, ENSURE study. Ann Oncol 2015;26:1883-9.

3. Maemondo M, Inoue A, Kobayashi K, et al. Gefitinib or chemotherapy for non-small-cell lung canScer with mutated EGFR. N Engl J Med 2010;362:2380-8.

4. Rosell R, Carcereny E, Gervais R, et al. erlotinib versus standard chemotherapy as first-line treatment for European patients with advanced EGFR mutation-positive non-small-cell lung cancer (EURTAC): a multicentre, open-label, randomised phase 3 trial. Lancet Oncol 2012;13:239-46.

5. Zhou C, Wu YL, Chen G, et al. erlotinib versus chemotherapy as first-line treatment for patients with advanced EGFR mutation-positive non-small-cell lung cancer (OPTIMAL, CTONG-0802): a multicentre, open-label, randomised, phase 3 study. Lancet Oncol 2011;12:735-42.

6. Mitsudomi T, Morita S, Yatabe Y, et al. Gefitinib versus cisplatin plus docetaxel in patients with non-small-cell lung cancer harbouring mutations of the epidermal growth factor receptor (WJTOG3405): an open label, randomised phase 3 trial. Lancet Oncol 2010;11:121-8.

7. Sequist LV, Yang JC, Yamamoto N, et al. Phase III study of afatinib or cisplatin plus pemetrexed in patients with metastatic lung adenocarcinoma with EGFR mutations. J Clin Oncol 2013;31:3327-34.

8. Wu YL, Zhou C, Hu CP, et al. Afatinib versus cisplatin plus gemcitabine for first-line treatment of Asian patients with advanced non-small-cell lung cancer harbouring EGFR mutations (LUX-Lung 6): an open-label, randomised phase 3 trial. Lancet Oncol 2014;15:213-22.

9. Soria JC, Ohe Y, Vansteenkiste J, et al. Osimertinib in Untreated EGFR-Mutated Advanced Non-Small-Cell Lung Cancer. N Engl J Med 2018;378:113-25.

10. Byers LA, Heymach JV. Dual targeting of the vascular endothelial growth factor and epidermal growth factor receptor pathways: rationale and clinical applications for non-small-cell lung cancer. Clin Lung Cancer 2007;8 Suppl 2:S79-85.
11. Ichihara E, Ohashi K, Takigawa N, et al. Effects of vandetanib on lung adenocarcinoma cells harbouring epidermal growth factor receptor T790M mutation in vivo. Cancer Res 2009;69:5091-8.

12. Saito H, Fukuhara T, Furuya N, et al. Erlotinib plus bevacizumab versus erlotinib alone in patients with EGFR-positive advanced non-squamous non-small-cell lung cancer (NEJ026): interim analysis of an open-label, randomised, multicentre, phase 3 trial. Lancet Oncol 2019;20:625-35.

13. Seto T, Kato T, Nishio M, et al. erlotinib alone or with bevacizumab as first-line therapy in patients with advanced non-squamous non-small-cell lung cancer harbouring EGFR mutations (JO25567): an open-label, randomised, multicentre, phase 2 study. Lancet Oncol 2014;15:1236-44.

14. Viloria-Petit A, Crombet T, Jothy S, et al. Acquired resistance to the antitumor effect of epidermal growth factor receptor-blocking antibodies in vivo: a role for altered tumor angiogenesis. Cancer Res 2001;61:5090-101.

15. Watanabe H, Ichihara E, Kayatani H, et al. Abstract 2131: Significant combination benefit of anti-VEGFR antibody and oncogene-targeted agents in EGFR or ALK mutant NSCLC cells. Atlanta, GA: AACR Annual Meeting 2019, March 29 to April 3, 2019.

16. Zhou K, Zhao S, Guo W, et al. efficacy and safety of erlotinib combined with bevacizumab in the treatment of non-small cell lung cancer: A systematic review and metaanalysis. Medicine (Baltimore) 2020;99:e18771.

17. Moher D, Liberati A, Tetzlaff J, et al. Preferred reporting items for systematic reviews and meta-analyses: the PRISMA statement. Ann Intern Med 2009;151:264-9, W64.

18. Ciuleanu T, Tsai CM, Tsao CJ, et al. A phase II study of erlotinib in combination with bevacizumab versus chemotherapy plus bevacizumab in the first-line treatment of advanced non-squamous non-small cell lung cancer.

Lung Cancer 2013;82:276-81.

19. Thomas M, Fischer J, Andreas S, et al. Erlotinib and bevacizumab versus cisplatin, gemcitabine and bevacizumab in unselected nonsquamous non-small cell lung cancer. Eur Respir J 2015;46:219-29.

20. Herbst RS, O'Neill VJ, Fehrenbacher L, et al. Phase II study of efficacy and safety of bevacizumab in combination with chemotherapy or erlotinib compared with chemotherapy alone for treatment of recurrent or refractory non-small cell lung cancer. J Clin Oncol 2007;25:4743-50.

21. Johnson BE, Kabbinavar F, Fehrenbacher L, et al. ATLAS: randomised, double-blind, placebo-controlled, 
phase IIIB trial comparing bevacizumab therapy with or without erlotinib, after completion of chemotherapy, with bevacizumab for first-line treatment of advanced nonsmall-cell lung cancer. J Clin Oncol 2013;31:3926-34.

22. Stinchcombe TE, Jänne PA, Wang X, et al. Effect of Erlotinib Plus Bevacizumab vs Erlotinib Alone on Progression-Free Survival in Patients With Advanced EGFR-Mutant Non-Small Cell Lung Cancer: A Phase 2 Randomized Clinical Trial. JAMA Oncol 2019;5:1448-55.

23. Nakagawa K, Garon EB, Seto T, et al. Ramucirumab plus erlotinib in patients with untreated, EGFRmutated, advanced non-small-cell lung cancer (RELAY): a randomised, double-blind, placebo-controlled, phase 3 trial. Lancet Oncol 2019;20:1655-69.

24. Zhou Q, Wu YL, Cheng Y, et al. 1480 O - CTONG 1509: Phase III study of bevacizumab with or without erlotinib in untreated Chinese patients with advanced EGFR-mutated NSCLC. Ann Oncol 2019;30:v603.

25. Herbst RS, Ansari R, Bustin F, et al. Efficacy of bevacizumab plus erlotinib versus erlotinib alone in advanced non-

Cite this article as: Chen Z, Jiang S, Li X, Zhang J, Liu L, Li J, Cao P, Xin Y, Zhang L. Efficacy and safety of anti-angiogenic drugs combined with erlotinib in the treatment of advanced non-small cell lung cancer: a meta-analysis of randomized clinical trials. Ann Palliat Med 2021;10(3):2687-2698. doi: 10.21037/ apm-20-1621 small-cell lung cancer after failure of standard first-line chemotherapy (BeTa): a double-blind, placebo-controlled, phase 3 trial. Lancet 2011;377:1846-54.

26. Jüni P, Altman DG, Egger M. Assessing the quality of controlled clinical trials. BMJ 2001;323:42-6.

27. Higgins JP, Altman DG, Gotzsche PC, et al. The Cochrane Collaboration's tool for assessing risk of bias in randomised trials. BMJ 2011;343:d5928.

28. Rosell R, Moran T, Queralt C, et al. Screening for epidermal growth factor receptor mutations in lung cancer. N Engl J Med 2009;361:958-67.

29. Yun CH, Mengwasser KE, Toms AV, et al. The T790M mutation in EGFR kinase causes drug resistance by increasing the affinity for ATP. Proc Natl Acad Sci USA 2008;105:2070-5.

30. Sos ML, Rode HB, Heynck S, et al. Chemogenomic profiling provides insights into the limited activity of irreversible EGFR Inhibitors in tumor cells expressing the T790M EGFR resistance mutation. Cancer Res 2010;70:868-74. 